\title{
REALIZATION OF ANALYSIS INTO EXPLICIT MATHEMATICS
}

\author{
SERGEI TUPAILO
}

\begin{abstract}
We define a novel interpretation $\mathscr{B}$ of second order arithmetic into Explicit Mathematics. As a difference from standard $\mathscr{O}$-interpretation. which was used before and was shown to interpret only subsystems proof-theoretically weaker than $\mathbf{T}_{0}$. our interpretation can reach the full strength of $\mathbf{T}_{0}$. The $\mathscr{R}$-interpretation is an adaptation of Kleene's recursive realizability. and is applicable only to intuitionistic theories.
\end{abstract}

Introduction. Systems of Explicit Mathematics were introduced by S. Feferman in the 70s as a logical framework for Bishop-style constructive mathematics (see [5], [6]). In [6] he gave an embedding of the basic theory $\mathbf{T}_{0}$ into a subsystem $\Delta_{2}^{1}-\mathbf{C A}+\mathbf{B I}$ of second order arithmetic and conjectured that the converse also holds. In [10] G. Jäger carried out a necessary well-ordering proof in $\mathbf{T}_{0}$, which together with [13] completed its proof-theoretical analysis and established proof-theoretic equivalence of the system of Explicit Mathematics $\mathbf{T}_{0}$, system of analysis $\Delta_{2}^{1}-\mathbf{C A}+\mathbf{B I}$. and the set theory KPi. However, up to now, there were no direct embeddings of strong conventional theories, e.g., analysis or set theory of the strength of $\mathbf{T}_{0}$ and higher, into Explicit Mathematics. This also yielded that the only method for establishing proof-theoretic lower bounds for $\mathbf{T}_{0}$ and stronger systems of Explicit Mathematics remained to be well-ordering proofs carried out directly in those theories.

The situation is quite different with Martin-Löf type theories, where, in addition to well-ordering proofs (see [16]), we also have direct embeddings of constructive set theory CZF, [1], and its extensions, [2, 14], or a subsystem of analysis IARI, [9]. The possibility of such an embedding is often considered as an evidence for constructivity of a given theory. The obstacle for similar embeddings into Explicit Mathematics was its specific nature, where intuitionistic and classical principles, set-theoretic and recursion-theoretic intuition can be combined. It is sufficiently straightforward to do for "weak" theories (essentially up to $\mathbf{\Pi}_{1}^{1}-\mathbf{C A}$ ): however, for stronger systems with mathematical meaning, where adding the law of excluded middle often results in dramatic increase in proof-theoretic strength (see, e.g., [15]), the distinction classical/intuitionistic must have played a prominent role. The price for this universality of Explicit Mathematics is that, while in ML type theories derivability simply means Kleene-type realizability, in EM these notions are different. The reason for this is that a lot of realizable formulas, e.g., Church's thesis and

Received October 9, 1999; accepted September 29, 2000.

Research supported by the Swiss National Science Foundation.

(C) 2001. Association for Symbolic Logic $0022-4812 / 01 / 6604-0022 / \$ 2.70$ 
axiom of choice in analysis, are incompatible with classical logic without damaging consistency or consistency strength.

In this paper we develop a realizability interpretation into Explicit Mathematics. We have chosen here the simplest example, realization of analysis, which keeps the amount of technical details at minimum. and demonstrates the method most distinctly. For constructive set theory CZF and its extensions, one combines realizability with other methods of interpreting set theory. This is reserved for another publication ([20]).

The paper is organized as follows. For reader's convenience in Sections 1 and 2 we briefly introduce the theory $\mathbf{T}_{0}$ and subsystems of analysis we are interested in. In Section 3 we define two interpretations of analysis into $\mathbf{T}_{0}$, a direct interpretation $\mathscr{D}$ and a realizability interpretation $\mathscr{R}$. A direct interpretation $\mathscr{D}$ means simply that variables are interpreted as ranging over natural numbers and sets of natural numbers and the meaning of logical connectives does not change. It's this interpretation which was used before, e.g., in [6. Ch. V] and [4. Ch. II. §1]. The drawback of this translation is that it does not really exploit the axiom of Join of Explicit Mathematics, the consequence of which being that the only systems which have been interpreted via $\mathscr{D}$ are proof-theoretically weaker than $\mathbf{T}_{0}$.

Alternatively, we define a realizability interpretation $\mathscr{R}$. which is a variant of Kleene 1945 recursive realizability. The general setting for realizing one language into another was given already in [6]; however, that paper studies in detail only realization of Explicit Mathematics into itself. As to relationships between the interpretations $\mathscr{D}$ and $\mathscr{R}$. we prove that they are equivalent over an applicative part App of $\mathbf{T}_{0}$ for first-order negative formulas, Theorem 2 , and $\mathscr{R}(F)$ implies $\mathscr{D}(F)$ for $F$ from a certain CC-class, Theorem 3 . Thus $\mathscr{R}$-interpretation automatically transfers proof-theoretic upper bounds from Explicit Mathematics to analysis, and lower bounds vice versa. Axiom of Choice, on the contrary, is an example of a formula for which $\mathscr{D}$ does not follow from $\mathscr{R}$, and is much stronger in presence of the law of excluded middle, Theorem 6 . In Section 4 we finally build realizations of various axioms, giving together the theory IARI of [9], which has the same proof-theoretic strength as $\mathbf{T}_{0}$.

Acknowledgements. I am grateful to Prof. Gerhard Jäger and Dr. Thomas Strahm for introducing me to the world of Explicit Mathematics.

§1. Explicit mathematics. The theory $\mathbf{T}_{0}$. We follow essentially the original typefree two-sorted formulation of Explicit Mathematics from [5]. Alternative formulations are given in [3] and [11].

Language $\mathscr{L}_{\mathbf{E M}}$. The theory $\mathbf{T}_{0}$ is formulated in a two-sorted language: operations (individuals) and names (classifications). Names are thought of as a special kind of operations, coding sets of operations. We use variables $a, b, c, \ldots$ as ranging over operations, and $\alpha, \beta, \gamma, \ldots$ as ranging over names. The operation constants of the theory are the following: combinators $k, s$, pairing $p$ and projections $p_{0}, p_{1}$, zero 0 , successor $s_{N}$ and predecessor $p_{N}$, distinction by cases on natural numbers $d_{N}$, join $\mathrm{j}$ and inductive generation $\mathrm{i}$. Additionally we have the following nine operation constants called name generators: nat, id, inv, emp, and, or, imp, all, ex. Terms are built from variables and constants by the following application clause: if $s$ and $t$ are 
terms then $s \cdot t$ is a term, so that the application function symbol $\cdot$ accepts arguments of both sorts and returns an operation. Atomic formulas are $s=t$ ( $s$ coincides with $t$ ) and $s \varepsilon t$ ( $s$ belongs to the set named by $t, s$ is classified under $t$ ), where $s$ and $t$ are terms. Formulas are built from atomic formulas by $\wedge, \vee, \rightarrow$ and two types of quantifiers, over operations and over names, e.g., $\forall a, \exists a, \forall \alpha, \exists \alpha$. Finally, expression is a term or a formula.

Abbreviations. We use the following standard abbreviations:

- $\neg F: \Leftrightarrow F \rightarrow \perp$;

- $F_{0} \leftrightarrow F_{1}: \Leftrightarrow\left(F_{0} \rightarrow F_{1}\right) \wedge\left(F_{1} \rightarrow F_{0}\right)$;

- $t \downarrow: \Leftrightarrow \exists x(t=x)$;

- $\mathscr{N}[t]: \Leftrightarrow \exists \alpha(t=\alpha)$;

- $s \simeq t: \Leftrightarrow(s \downarrow \vee t \downarrow) \rightarrow s=t$

- $s \dot{\subset} t: \Leftrightarrow \forall x \in s(x \in t) ; s \doteq t: \Leftrightarrow s \dot{\subset} t \wedge t \dot{\subset} s$;

- $r: s \mapsto t$ for $\forall x \in s(r x \in t)$;

- $r: s^{1} \mapsto t$ for $r: s \mapsto t, r: s^{m+1} \mapsto t$ for $\forall x \varepsilon s\left(r x: s^{m} \mapsto t\right)$;

- $t^{\prime}$ for $\mathrm{s}_{\mathrm{N}} \cdot t ; 1$ for $0^{\prime} ; s t$ for $s \cdot t ; t\left(s_{1}, \ldots, s_{n}\right)$ for $\left(\ldots\left(t s_{1}\right) \ldots s_{n}\right) ;\langle s, t\rangle$ for $(\mathrm{p} s) t$; $s \neq t$ for $\neg s=t$, etc.

\section{Syntactical conventions.}

1. We use $e[*]$ for an expression $e$, possibly containing occurrences of a variable $*$ (of appropriate sort). In this context by $e[t]$ we mean the result of substituting expression $t$ for all occurrences of $*$ in $e$.

2. Parentheses in terms are assumed to be associated to the left: e.g., $s \cdot t \cdot u$ is read as $(s \cdot t) \cdot u$.

3. We adopt the following priority among propositional connectives and their abbreviations: $\neg, \wedge, \vee, \rightarrow, \leftrightarrow$. For example, $F_{1} \vee \neg F_{2} \wedge F_{3} \rightarrow F_{4} \leftrightarrow F_{5}$ has to be read as $\left(\left(F_{1} \vee\left(\left(\neg F_{2}\right) \wedge F_{3}\right)\right) \rightarrow F_{4}\right) \leftrightarrow F_{5}$.

Logic. Intuitionistic 2 -sorted logic of partial terms with equality. See, e.g., [3, Ch. VI, 1] or $[18,1.3]$. We take $\perp$ (falsity) as a propositional constant with standard axioms pertaining to it.

Axioms. The axioms are divided in six groups, according to their nature.

I. Applicative axioms. These axioms formalize that operations form a partial combinatory algebra, that we have pairing and projections, usual closure conditions on natural numbers, as well as definition by numerical cases:

(1) $\mathrm{k} a b=a$;

(2) $s a b \downarrow \wedge s a b c \simeq a c(b c)$;

(3) $\mathrm{p} a b \downarrow \wedge \mathrm{p}_{0} a \downarrow \wedge \mathrm{p}_{1} a \downarrow \wedge \mathrm{p}_{0}(\mathrm{p} a b)=a \wedge \mathrm{p}_{1}(\mathrm{p} a b)=b$;

(4) $0 \varepsilon$ nat $\wedge \forall x \varepsilon$ nat ( $\mathrm{s}_{\mathrm{N}} x \varepsilon$ nat);

(5) $\forall x \varepsilon$ nat $\left(\mathrm{s}_{\mathrm{N}} x \neq 0 \wedge \mathrm{p}_{\mathrm{N}}\left(\mathrm{s}_{\mathrm{N}} x\right)=x\right)$;

(6) $\forall x \varepsilon$ nat $\left(x \neq 0 \rightarrow \mathrm{p}_{\mathrm{N}} x \varepsilon\right.$ nat $\left.\wedge \mathrm{s}_{\mathrm{N}}\left(\mathrm{p}_{\mathrm{N}} x\right)=x\right)$;

(7) $a \varepsilon$ nat $\wedge b \varepsilon$ nat $\rightarrow\left(a=b \rightarrow \mathrm{d}_{N} x y a b=x\right) \wedge\left(a \neq b \rightarrow \mathrm{d}_{N} x y a b=y\right)$.

\section{Induction on nat.}

$$
\varphi[0] \wedge \forall x\left(\varphi[x] \rightarrow \varphi\left[\mathrm{s}_{\mathrm{N}} x\right]\right) \rightarrow \forall x \varepsilon \operatorname{nat} \varphi[x]
$$

for each formula $\varphi$. 
The following lemmas 1.1 and 1.2 are provable using only applicative axioms I; Lemma 1.3 in addition calls for induction on natural numbers II (see, for example, [6], [3], or a review [12]).

Lemma 1.1 ( $\lambda$-abstraction). For every term $t[x]$ there exists a term $\lambda x . t[x]$ such that $\lambda x . t[x] \downarrow$ and for every term $s$

$$
s \downarrow \rightarrow(\lambda x . t[x]) s \simeq t[s]) .
$$

Lemma 1.2 (Recursion Theorem). There exists a closed term rec such that

$$
\operatorname{rec} f \downarrow \wedge \operatorname{rec} f x \simeq f(\operatorname{rec} f) x .
$$

Lemma 1.3 (Primitive recursion on natural numbers). There exists a closed term prim such that

$$
\begin{aligned}
& f: \text { nat } \mapsto \text { nat } \wedge g: \text { nat }^{3} \mapsto \text { nat } \wedge x \varepsilon \text { nat } \wedge y \varepsilon \text { nat } \longrightarrow \\
& \quad \operatorname{prim} f g: \text { nat }^{2} \mapsto \text { nat } \wedge \operatorname{prim} f g x 0=f x \wedge \operatorname{prim} f g x\left(\mathrm{~s}_{N} y\right)=g x y(\operatorname{prim} f g x y) .
\end{aligned}
$$

III. Explicit representation. This axiom states that each name is an operation:

$$
\exists x(x=\alpha) .
$$

IV. Elementary comprehension (ECA). These axiomatize name generators:

(1) $\mathscr{N}$ [nat];

(2) $\mathscr{N}\left[\right.$ id] $\wedge \forall x\left(x \varepsilon\right.$ id $\left.\leftrightarrow x=\left\langle\mathrm{p}_{0} x, \mathrm{p}_{1} x\right\rangle \wedge \mathrm{p}_{0} x=\mathrm{p}_{1} x\right)$;

(3) $\mathscr{N}[\operatorname{inv}(f, \alpha)] \wedge \forall x(x \varepsilon \operatorname{inv}(f, \alpha) \leftrightarrow f x \varepsilon \alpha)$;

(4) $\mathscr{N}[\mathrm{emp}] \wedge \forall x(x \in \mathrm{emp} \leftrightarrow \perp)$;

(5) $\mathcal{N}[$ and $(\alpha, \beta)] \wedge \forall x(x \varepsilon$ and $(\alpha, \beta) \leftrightarrow x \varepsilon \alpha \wedge x \varepsilon \beta)$;

(6) $\mathcal{N}[\operatorname{or}(\alpha, \beta)] \wedge \forall x(x \varepsilon \operatorname{or}(\alpha, \beta) \leftrightarrow x \varepsilon \alpha \vee x \varepsilon \beta)$;

(7) $\mathcal{N}[\operatorname{imp}(\alpha, \beta)] \wedge \forall x(x \varepsilon \operatorname{imp}(\alpha, \beta) \leftrightarrow x \varepsilon \alpha \rightarrow x \varepsilon \beta)$;

(8) $\mathscr{N}[$ all $\alpha] \wedge \forall x(x \varepsilon$ all $\alpha \leftrightarrow \forall y(\langle x, y\rangle \varepsilon \alpha))$;

(9) $\mathscr{N}[\operatorname{ex} \alpha] \wedge \forall x(x \varepsilon \operatorname{ex} \alpha \leftrightarrow \exists y(\langle x, y\rangle \varepsilon \alpha))$.

DeFinition 1.1 (Elementary formula). A formula is elementary if and only if it is constructed from $s=t$ and $t \varepsilon \alpha$ by means of $\wedge, \vee, \rightarrow, \forall x, \exists x$ only. (No occurrences of $t \varepsilon s$ with $s$ not a name variable and name quantifiers are allowed.)

The following lemma is an intuitionistic analogue of reducing Elementary Comprehension as stated in [5] to name generators nat, id, co, int, dom and inv, which holds in classical setting (see [7]); its proof requires only axioms I, III and IV. For alternative intuitionistic reductions of Elementary Comprehension to a finite number of its instances see [9, Sect. 1] and [17, Sect. 3].

Lemma 1.4 (ECA). If a formula $F:=F[x ; \bar{a} ; \bar{\alpha}]$ is elementary then there exists a term $\mathrm{t}_{F}^{x}$ such that $\mathrm{FV}\left(\mathrm{t}_{F}^{x}\right)=\mathrm{FV}(F) \backslash\{x\}$ and

$$
\mathcal{N}\left[\mathrm{t}_{F}^{x}\right] \wedge \forall x\left(x \varepsilon \mathrm{t}_{F}^{x} \leftrightarrow F\right)
$$


Proof. The term $t_{F}^{x}$ is built by recursion on $F$ :

$$
\mathrm{t}_{F}^{x}:= \begin{cases}\operatorname{inv}(\lambda x .\langle s[x], t[x]\rangle, \text { id }) & \text { if } F \text { is } s[x]=t[x] ; \\ \operatorname{inv}(\lambda x . s[x], \alpha) & \text { if } F \text { is } s[x] \varepsilon \alpha: \\ \operatorname{emp} & \text { if } F \text { is } \perp ; \\ \operatorname{and}\left(\mathrm{t}_{F_{0}[x]}^{x}, \mathrm{t}_{F_{1}[x]}^{x}\right) & \text { if } F \text { is } F_{0}[x] \wedge F_{1}[x] ; \\ \operatorname{or}\left(\mathrm{t}_{F_{0}[x]}^{x}, \mathrm{t}_{F_{1}[x]}^{x}\right) & \text { if } F \text { is } F_{0}[x] \vee F_{1}[x] ; \\ \operatorname{imp}\left(\mathrm{t}_{F_{0}[x]}^{x}, \mathrm{t}_{F_{1}[x]}^{x}\right) & \text { if } F \text { is } F_{0}[x] \rightarrow F_{1}[x] ; \\ \operatorname{allt}_{G_{\left[p_{0} z p_{1} z\right]}^{z}}^{z} & \text { if } F \text { is } \forall y G[x, y] \\ \operatorname{ext}_{G\left[\mathrm{p}_{0} z \mathrm{p}_{1} z\right]}^{z} & \text { if } F \text { is } \exists y G[x, y] .\end{cases}
$$

Now the property of $\mathrm{t}_{F}^{x}$ is proved by induction on $F$.

V. Join $(\mathbf{J})$. This axiom states that if $f$ is an operation from a set named by $\alpha$, each value of which is a name, then $\mathrm{j}(\alpha, f)$ names a disjoint union of all $f x$ for $x \varepsilon \alpha$ :

$$
\begin{aligned}
\forall x \varepsilon \alpha \mathscr{N}[f x] \rightarrow(\mathcal{N}[\mathrm{j}(\alpha, f)] \wedge \forall z(z \varepsilon \mathrm{j}(\alpha, f) & \\
& \leftrightarrow \exists x \varepsilon \alpha \exists y(z=\langle x, y\rangle \wedge y \varepsilon f x))) .
\end{aligned}
$$

VI. Inductive Generation (IG). The first part of this axiom states that i $(\alpha, \beta)$ names a well-founded part of a set named by $\alpha$ along an ordering named by $\beta$; the second part allows induction over that set for an arbitrary formula:

$$
\begin{aligned}
\mathscr{N}[\mathrm{i}(\alpha, \beta)] & \wedge \forall x \varepsilon \alpha(\forall y(\langle y, x\rangle \varepsilon \beta \rightarrow y \varepsilon \mathrm{i}(\alpha, \beta)) \rightarrow x \varepsilon \mathrm{i}(\alpha, \beta))) \\
& \wedge(\forall x \varepsilon \alpha(\forall y(\langle y, x\rangle \varepsilon \beta \rightarrow \phi[y]) \rightarrow \phi[x]) \rightarrow \forall x \varepsilon \mathrm{i}(\alpha, \beta)) \phi[x]),
\end{aligned}
$$

where $\phi \in \mathscr{L}_{\mathrm{EM}}$ is an arbitrary formula.

The theory App is the one containing only applicative axioms I; EON has axioms I-II. The theory EONN has axioms of the groups I-III. EET is EONN + ECA, EETJ is EET + J and $\mathbf{T}_{0}$ is EETJ + IG. $^{1}$

By TND (tetrium non datur), both in Explicit Mathematics and analysis, we mean a schema consisting of all instances of the Law of Excluded Middle.

§2. Subsystems of analysis. The basic theory EHA (Elementary Heyting Analysis) is formulated in a two-sorted language $\mathscr{L}_{2}$ : numbers and sets of numbers. We use variables $a, b, c, \ldots$ as ranging over numbers, and $A, B, C, \ldots$ as ranging over sets. There is only one individual constant 0 . The function constants are: successor ', pairing $(*, *)$ and projections $(*)_{0},(*)_{1}$, and also countably many function constants $f_{1}, f_{2}, \ldots$ for primitive recursive functions. Terms are built as usual. Atomic formulas are of the kinds $s=t$ and $s \in A$ ( $s$ and $t$ are terms). Formulas are built from atomic formulas by $\wedge, \vee, \rightarrow$ and two types of quantifiers, over numbers and over sets, e.g., $\forall x, \exists x, \forall X, \exists X$. By FV(e) we denote the set of free variables occurring in an expression $e$, and by $\mathrm{FV}_{0}(e)$ and $\mathrm{FV}_{1}(e)$ respectively the set of first

\footnotetext{
${ }^{1}$ In the literature the names EET and EETJ are also used for theories as defined here, but with restricted induction $I I$.
} 
and second order free variables of $e$. A formula is called negative if and only if it doesn't contain $\vee$ or $\exists$. A formula is first-order if and only if it doesn't contain second-order variables. A formula is arithmetical if and only if it does not contain second-order quantifiers. We use the same abbreviations and syntactical conventions as in the previous Section. The logic is intuitionistic 2-sorted logic. Axioms are the following: equality axioms, Peano axioms, prim.-rec. definitions for function symbols $(*, *),(*)_{0},(*)_{1}, f_{1}, f_{2}, \ldots$ and mathematical induction schema. Note that we have no comprehension in EHA. thus EHA being a conservative extension of Heyting arithmetic.

EHA is the basic theory of analysis in this paper. Additionally, we will consider extensions of EHA by the following axioms.

Arithmetic comprehension (ACA):

$\exists X \forall x(x \in X \leftrightarrow \psi[x])$ for $\psi$ arithmetical.

Axiom of Choice (AC):

$\forall x \exists Y \phi[x, Y] \rightarrow \exists Z \forall x \phi\left[x, Z_{x}\right]$ for all formulas $\phi$.

\section{Replacement (RP):}

$\forall X\left(\forall x \in X \exists ! Y \phi[x, Y] \rightarrow \exists Z \forall x \in X \phi\left[x, Z_{x}\right]\right)$ for all formulas $\phi$, where $\phi\left[x, Z_{x}\right]$ arises from $\phi[x, Z]$ by replacing each occurrence of $s \in Z$ by $(x, s) \in Z$.

Inductive Generation (IGA):

$\forall X \forall Y \exists Z\left(\mathrm{WP}_{Y}[X, Z] \wedge \operatorname{TI}_{Y}[X, Z, \phi]\right)$ for all formulas $\phi$, where we adopt the following abbreviations:

$$
\begin{array}{llll}
\mathrm{WP}_{Y}[X, Z] & \text { denotes } & \operatorname{Prog}_{Y}[X, Z] \wedge \forall U\left(\operatorname{Prog}_{Y}[X, U] \rightarrow Z \subseteq U\right) \\
\operatorname{Prog}_{Y}[X, Z] & \text { denotes } \forall x \in X(\forall y(y<Y \rightarrow \rightarrow y \in Z) \rightarrow x \in Z) \\
\operatorname{Prog}_{Y}[X, \phi] & \text { denotes } & \forall x \in X(\forall y(y<Y x \rightarrow \phi[y]) \rightarrow \phi[x]) \\
\operatorname{TI}_{Y}[X, Z, \phi] & \text { denotes } & \operatorname{Prog}_{Y}[X, \phi] \rightarrow \forall z \in Z \phi[z] \\
y<_{Y} x & \text { denotes } & (y, x) \in Y .
\end{array}
$$

IARI of [9] is the theory EHA + ACA + RP + IGA. It's shown there that IARI is directly interpretable in Martin-Löf type theory $\mathbf{M L}_{\mathbf{1 w}}$ and has the same prooftheoretic strength as $\mathbf{M L}_{1 \mathrm{w}}$ and $\mathbf{T}_{0}$.

§3. Interpretations into Explicit Mathematics. In this section we define two interpretations of analysis into Explicit Mathematics, a direct interpretation $\mathscr{D}$ and a realizability interpretation $\mathscr{R}$, and study relationships between them.

First, for each individual and function constant $f \in \mathscr{L}_{2}$ by Lemma 1.3 we can define an operation $\mathrm{N}(f)$ presenting the same primitive-recursive function as $f$ and having the following property: if $n$ is the arity of $f$ then EET proves

$$
\bigwedge_{i=1}^{n} x_{i} \varepsilon \text { nat } \rightarrow \mathrm{N}(f) x_{1} \ldots x_{n} \varepsilon \text { nat. }
$$

We may assume that $\mathrm{N}(0)$ is 0 and $\mathrm{N}\left(\mathrm{s}_{\mathrm{N}}\right)$ is ${ }^{\prime}$. Now terms of $\mathscr{L}_{2}$ are translated as follows: 
DEFINITION $3.1(\mathrm{~N}(t))$.

$$
\left\{\begin{array}{l}
\mathrm{N}(x):=x ; \\
\mathrm{N}\left(f t_{1} \ldots t_{n}\right):=\mathrm{N}(f) \mathrm{N}\left(t_{1}\right) \ldots \mathrm{N}\left(t_{n}\right) .
\end{array}\right.
$$

For each second-order variable $A \in \mathscr{L}_{2}$ we assume a name variable $\alpha_{A} \in \mathscr{L}_{\mathrm{EM}}$. A direct interpretation $\mathscr{D}: \mathscr{L}_{2} \mapsto \mathscr{L}_{\mathrm{EM}}$ was introduced in [6] and used later on (see, for example, [4, Ch. II] and [8]). It is defined as follows:

DEFINITION 3.2 ( $\mathscr{D}$-interpretation).

$$
\begin{cases}\mathscr{D}(s=t):=\mathrm{N}(s)=\mathrm{N}(t) & \\ \mathscr{D}(t \in A):=\mathrm{N}(t) \varepsilon \alpha_{A} & \\ \mathscr{D}\left(F_{0} \circ F_{1}\right):=\mathscr{D}\left(F_{0}\right) \circ \mathscr{D}\left(F_{1}\right), & \text { for } \circ \in\{\wedge, \vee, \rightarrow\} \\ \mathscr{D}(Q x G):=Q x \varepsilon \text { nat } \mathscr{D}(G), & \text { for } Q \in\{\forall, \exists\} \\ \mathscr{D}(Q X G):=Q \alpha_{X} \subset \text { nat } \mathscr{D}(G), & \text { for } Q \in\{\forall, \exists\} .\end{cases}
$$

The following lemma is straightforward (see [4, Ch. II, $\S 1]$ ):

LEMMA 3.1. For each theorem $F$ of the theory EHA $+\mathbf{A C A} \mathscr{D}(F)$ is provable in EET.

Alternatively, we define a realizability interpretation $\mathscr{R}: \mathscr{L}_{2} \mapsto \mathscr{L}_{\mathrm{EM}}$.

Definition $3.3\left(\mathscr{N}^{\mathrm{N}}\right) . \mathscr{N}^{\mathrm{N}}[t]:=\mathscr{N}[t] \wedge \forall z \varepsilon t\left(z=\left\langle\mathrm{p}_{0} z, \mathrm{p}_{1} z\right\rangle \wedge \mathrm{p}_{0} z \varepsilon\right.$ nat $)$.

DEFINITION $3.4\left(\mathfrak{r}\right.$ realizes $F, \mathfrak{r} \mathbf{r n} F$ ). For each formula $F \in \mathscr{L}_{2}$ we define a formula $\mathfrak{r} \mathbf{r n} F \in \mathscr{L}_{\text {EM }}$. $\mathfrak{r}$ will always be treated as a new free individual variable. The definition is given by the table below:

$\begin{array}{ll}F & \mathfrak{r} \mathbf{r n} F \\ \perp & \perp \\ s=t & \mathrm{~N}(s)=\mathrm{N}(t) \\ t \in A & \langle\mathrm{~N}(t), \mathfrak{r}\rangle \varepsilon \alpha_{A} \\ F_{0} \wedge F_{1} & \mathrm{p}_{0} \mathfrak{r} \mathbf{r n} F_{0} \wedge \mathrm{p}_{1} \mathfrak{r} \mathbf{r n} F_{1} \\ F_{0} \vee F_{1} & \mathrm{p}_{0} \mathfrak{r} \varepsilon \text { nat } \wedge\left(\mathrm{p}_{0} \mathfrak{r}=0 \rightarrow \mathrm{p}_{1} \mathfrak{r} \mathbf{r n} F_{0}\right) \wedge \\ F_{0} \rightarrow F_{1} & \forall \mathfrak{r}\left(\mathfrak{r} \mathbf{r n} F_{0} \rightarrow \mathfrak{r} \downarrow \wedge \text { r } \mathfrak{r} \mathbf{r n} F_{1}\right) \\ \forall x G[x] & \forall x \varepsilon \text { nat }(\mathfrak{r} x \downarrow \wedge \mathfrak{r} x \mathbf{r n} G[x]) \\ \exists x G[x] & \mathrm{p}_{0} \mathfrak{r} \varepsilon \operatorname{nat} \wedge \mathrm{p}_{1} \mathfrak{r} \mathbf{r n} G\left[\mathrm{p}_{0} \mathfrak{r}\right] \\ \forall X G[X] & \forall \alpha_{X}\left(\mathscr{N}^{\mathrm{N}}\left[\alpha_{X}\right] \rightarrow \mathfrak{r} \alpha_{X} \downarrow \wedge \mathfrak{r} \alpha_{X} \mathbf{r n} G\left[\alpha_{X}\right]\right) \\ \exists X G[X] & \mathcal{N}^{\mathrm{N}}\left[\mathrm{p}_{0} \mathfrak{r}\right] \wedge \mathrm{p}_{1} \mathfrak{r} \mathbf{r n} G\left[\mathrm{p}_{0} \mathfrak{r}\right]\end{array}$

REMARK. According to our notation for substitution, p. 1850, in the previous definition $\mathrm{p}_{1} \mathfrak{r} \mathbf{r n} G\left[\mathrm{p}_{0} \mathfrak{r}\right]$ in the last clause, for example, stands for $(\mathfrak{r} \mathbf{r n} G[X])_{\mathfrak{r}, \alpha_{X}}^{\boldsymbol{p}_{1} \mathfrak{r}, p_{0} r}$.

DEFINITION 3.5 ( $\mathscr{R}$-interpretation). For each $F \in \mathscr{L}_{2}$ we define

$$
\mathscr{R}(F):=\exists \mathfrak{x}(\mathfrak{x} \mathbf{r n} F) \text {. }
$$


REMARK. An important difference of $\mathscr{R}$-interpretation from $\mathscr{D}$-interpretation is that sets are translated not as (names of) sets of natural numbers, but as (names of) sets of pairs, only first elements of which are natural numbers (see the clause for $t \in A$ ). This is a standard effect in realizability interpretations of analysis, see, e.g., [18, Sect. 7.2]. The second element $\mathfrak{r}$ of a pair $\langle N(t), \mathfrak{r}\rangle$ can be thought of as a "proof" that $t \in A$.

Syntactical convention. We will often use the Fraktur font $\mathfrak{a}, \mathfrak{b}, \mathfrak{c}, \ldots$ to stress that a given term plays a role as realization. Formally, this is not a new type of objects; it's just a substitution for $a, b, c, \ldots$ used for better readability.

Abbreviation. $\mathfrak{t} \downarrow \mathbf{r n} F$ will be used for $\mathfrak{t} \downarrow \wedge \mathfrak{t} \mathbf{r n} F$.

DEFINITION 3.6 (Realization, realizable).

1. A term $\mathfrak{t} \in \mathscr{L}_{\mathrm{EM}}$ is called realization for a formula $F \in \mathscr{L}_{2}$ in a theory $\mathscr{T} \in \mathscr{L}_{\mathrm{EM}}, \mathbf{A p p} \subset \mathscr{T}$, if and only if

$$
\mathrm{FV}(\mathfrak{t}) \subset \mathrm{FV}_{0}(F) \bigcup\left\{\alpha_{A} \mid A \in \mathrm{FV}_{1}(F)\right\}
$$

and

$$
\mathscr{T} \vdash \bigwedge_{a \in \mathrm{FV}_{0}(F)} a \varepsilon \text { nat } \wedge \bigwedge_{A \in \mathrm{FV}_{1}(F)} \mathscr{N}^{\mathrm{N}}\left[\alpha_{A}\right] \rightarrow \operatorname{trn} F
$$

2. If there exists such a term $t$ then $F$ is called realizable in $\mathscr{T}$. We call a theory $\mathrm{T}_{\mathrm{A}}$ realizable in $\mathscr{T}$ if and only if every theorem of $\mathrm{T}_{\mathrm{A}}$ is realizable in $\mathscr{T}$.

NOTE. If $F$ is closed and realizable in $\mathscr{T}$ then $\mathscr{T} \vdash \mathscr{R}(F)$.

THEOREM 1. Each theorem of EHA is realizable in EONN.

The proof is standard and can be found, for example, in [19, Ch. IV, Sect. 4].

NotE. According to Theorem 1, to prove realizability of a theory $\mathrm{T}_{\mathrm{A}} \in \mathscr{L}_{2}$, EHA $\subset \mathrm{T}_{\mathrm{A}}$, it is sufficient to construct realizing terms for additional axioms of $\mathrm{T}_{\mathrm{A}}$. This is what we do in Section 4.

Now we turn to the relationship between $\mathscr{D}$ and $\mathscr{R}$-interpretations. For firstorder negative formulas we can define canonical realizers as in [18, Lemma 1.10].

Definition 3.7 (Canonical realization, $\operatorname{can}_{F}$ ). For $F \in \mathscr{L}_{2}$ first-order negative we define a term $\operatorname{can}_{F} \in \mathscr{L}_{\mathrm{EM}}$ (canonical realization of $F$ ) in the following way:

$$
\operatorname{can}_{F}:= \begin{cases}0 & \text { if } F \text { is } s=t ; \\ \left\langle\operatorname{can}_{F_{0}}, \operatorname{can}_{F_{1}}\right\rangle & \text { if } F \text { is } F_{0} \wedge F_{1} ; \\ \lambda \mathrm{r} \cdot \operatorname{can}_{F_{1}} & \text { if } F \text { is } F_{0} \rightarrow F_{1} \\ \lambda x \cdot \operatorname{can}_{G[x]} & \text { if } F \text { is } \forall x G[x] .\end{cases}
$$

Note. For every $F \operatorname{can}_{F}$ is closed and App $\vdash \operatorname{can}_{F} \downarrow$.

TheOREM $2(\mathscr{D}(F) \leftrightarrow \mathscr{R}(F))$. For $F \in \mathscr{L}_{2}$ being first-order negative in App we have:

(i) $\exists \mathfrak{x}(\mathfrak{x} \mathbf{r n} F) \rightarrow \mathscr{D}(F)$;

(ii) $\mathscr{D}(F) \rightarrow \operatorname{can}_{F} \mathbf{r n} F$;

(iii) $\mathscr{D}(F) \leftrightarrow \mathscr{R}(F)$. 
Proof. We prove (i) and (ii) by simultaneous induction on $F$.

If $F$ is atomic then it is of the form $s=t$ and both $\mathscr{D}(F)$ and $\operatorname{trn} F$ are of the form $\mathrm{N}(s)=\mathrm{N}(t)$.

Assume $F$ is $F_{0} \wedge F_{1}$ and the claim holds for $F_{0}$ and $F_{1}$.

Assume $\mathfrak{r} \mathbf{r n}\left(F_{0} \wedge F_{1}\right)$. Then we have $\mathrm{p}_{0} \mathfrak{r} \mathbf{r n} F_{0} \wedge \mathrm{p}_{1} \mathfrak{r} \mathbf{r n} F_{1}$. By IH(i) we get $\mathscr{D}\left(F_{0}\right) \wedge \mathscr{D}\left(F_{1}\right)$. i.e.. $\mathscr{D}(F)$.

Assume $\mathscr{D}\left(F_{0} \wedge F_{1}\right)$. Then we have $\mathscr{D}\left(F_{0}\right) \wedge \mathscr{D}\left(F_{1}\right)$. By IH (ii) we get $\operatorname{can}_{F_{0}} \mathbf{r n} F_{0} \wedge$ $\operatorname{can}_{F_{1}} \mathbf{r n} F_{1}$. which by Definition 3.7 gives $\operatorname{can}_{F} \mathbf{r n} F$.

Assume $F$ is $F_{0} \rightarrow F_{1}$ and the claim holds for $F_{0}$ and $F_{1}$.

Assume $\mathfrak{r} \mathbf{r n}\left(F_{0} \rightarrow F_{1}\right)$ and assume $\mathscr{D}\left(F_{0}\right)$. By IH (ii) we have $\operatorname{can}_{F_{0}} \mathbf{r n} F_{0}$. Then r $\operatorname{can}_{F_{0}} \downarrow$ rn $F_{1}$ and by IH (i) $\mathscr{D}\left(F_{1}\right)$.

Assume $\mathscr{D}\left(F_{0} \rightarrow F_{1}\right)$ and assume $\mathfrak{r n} F_{0}$. By IH (i) $\mathscr{D}\left(F_{0}\right)$ and therefore $\mathscr{D}\left(F_{1}\right)$. By IH (ii) $\operatorname{can}_{F_{1}} \mathbf{r n} F_{1}$, which by Definition 3.7 gives $\operatorname{can}_{F} \mathbf{m n} F$.

Assume $F$ is $\forall x G[x]$ and the claim holds for $G[x]$.

Assume $\mathfrak{r} \mathbf{r n}(\forall x G[x])$. Then $\forall x \varepsilon$ nat $(\mathfrak{r} x \downarrow$ rn $G[x])$. By IH (i) this yields $\forall x \varepsilon$ nat $\mathscr{D}(G[x])$. i.e. $\mathscr{D}(F)$.

Assume $\mathscr{D}(\forall x G[x])$. Then $\forall x \varepsilon$ nat $\mathscr{D}(G[x])$. By IH (ii) this yields $\forall x \varepsilon$ nat $\left(\operatorname{can}_{G[x]} \mathbf{r n} G[x]\right)$, which by Definition 3.7 gives $\operatorname{can}_{F} \mathbf{r n} F$.

(iii) is an immediate consequence of (i) and (ii).

Definition 3.8 (CC-class, cf. [18. Sect. 1.14]). A first-order formula $F \in \mathscr{L}_{2}$ belongs to the CC-class if and only if for every subformula $G \rightarrow H$ of it $G$ is negative.

Theorem $3(\mathscr{R}(F) \rightarrow \mathscr{D}(F))$. If $F \in C C$ then App $\vdash \mathscr{R}(F) \rightarrow \mathscr{D}(F)$.

Proof. The claim is proved by induction on $F$.

If $F$ is atomic then it is of the form $s=t$ and both $\mathscr{D}(F)$ and $\operatorname{trn} F$ are of the form $\mathrm{N}(s)=\mathrm{N}(t)$.

Assume $F$ is $F_{0} \wedge F_{1}$ and $\mathfrak{r} \mathbf{r n}\left(F_{0} \wedge F_{1}\right)$. Then we have $\mathrm{p}_{0} \mathrm{r} \mathbf{r n} F_{0} \wedge \mathrm{p}_{1} \mathfrak{r} \mathbf{r n} F_{1}$. By IH we get $\mathscr{D}\left(F_{0}\right) \wedge \mathscr{D}\left(F_{1}\right)$. i.e., $\mathscr{D}(F)$.

Assume $F$ is $F_{0} \vee F_{1}$ and $\mathfrak{r} \mathbf{r n}\left(F_{0} \vee F_{1}\right)$. Then we have $\mathrm{p}_{0} \mathfrak{r} \varepsilon$ nat $\wedge\left(\mathrm{p}_{0} \mathfrak{r}=0 \rightarrow\right.$ $\left.\mathrm{p}_{1} \mathfrak{r} \mathbf{r n} F_{0}\right) \wedge\left(\mathrm{p}_{0} \mathfrak{r} \neq 0 \rightarrow \mathrm{p}_{1} \mathfrak{r} \mathbf{r n} F_{1}\right)$. In the case $\mathrm{p}_{0} \mathfrak{r}=0$ by IH $\mathscr{D}\left(F_{0}\right):$ if $\mathrm{p}_{0} \mathfrak{r} \neq 0$ then similarly $\mathscr{D}\left(F_{1}\right)$. In both cases $\mathscr{D}(F)$.

Assume $F$ is $F_{0} \rightarrow F_{1}$ and $\mathfrak{r} \mathbf{r n}\left(F_{0} \rightarrow F_{1}\right)$. Then $F_{0}$ is first-order negative. Assume $\mathscr{D}\left(F_{0}\right)$. By the previous Theorem (ii) $\operatorname{can}_{F_{0}} \mathbf{r n} F_{0}$. Then $\mathbf{r} \operatorname{can}_{F_{0}} \downarrow \mathbf{r n} F_{1}$. Now by IH $\mathscr{D}\left(F_{1}\right)$.

Assume $F$ is $\forall x G[x]$ and $\mathfrak{r} \mathbf{r n}(\forall x G[x])$. Then $\forall x \varepsilon$ nat $(\mathfrak{r} x \downarrow \mathbf{r n} G[x])$. By IH this yields $\forall x \varepsilon$ nat $\mathscr{D}(G[x])$, i.e., $\mathscr{D}(F)$.

Assume $F$ is $\exists x G[x]$ and $\mathfrak{r} \mathbf{r n}(\exists x G[x])$. Then we have $\mathrm{p}_{0} \mathrm{r} \varepsilon$ nat $\wedge \mathrm{p}_{1} \mathfrak{r} \mathbf{r n} G\left[\mathrm{p}_{0} \mathrm{r}\right]$. By IH $\mathscr{D}\left(G\left[\mathrm{p}_{0} \mathrm{r}\right]\right)$, which implies $\mathscr{D}(F)$.

Remarks about proof-theoretic strength. We assume here that $\mathrm{T}_{\mathrm{A}}$ is realizable in $\mathscr{T}$.

(1) Note that Consis $(\mathrm{T})$ is $\Pi_{1}^{0}$-formula for any theory $\mathrm{T} \in \mathscr{L}_{2}$ with a decidable predicate $\operatorname{Prf}_{T}(a, b)$. Therefore, if $\mathrm{T}_{\mathrm{A}} \vdash \operatorname{Consis}(\mathrm{T})$ then $\mathscr{T} \vdash \mathscr{D}(\operatorname{Consis}(\mathrm{T}))$.

(2) Note that prenex formulas, in particular $\Pi_{2}^{0}$-formulas. are CC. Therefore. if $\mathrm{T}_{\mathrm{A}}$ proves totality of a function $f$, then so does $\mathscr{T}$. 
(3) Let $f \prec$ be a characteristic function of a standard prim.-rec. ordering $\prec$ on natural numbers (e.g., an initial part of some fixed standard ordering), i.e.,

$$
y \prec x: \Leftrightarrow f_{\prec}(y, x)=0 .
$$

For every first-order negative formula $F[x] \in \mathscr{L}_{2}$ we set:

$$
\begin{aligned}
\operatorname{Prog}(\prec, F) & : \Leftrightarrow \forall x(\forall y(f \prec(y, x)=0 \rightarrow F[y]) \rightarrow F[x]), \\
\text { Fund }(\prec, F) & \Leftrightarrow \operatorname{Prog}(\prec, F) \rightarrow \forall x F[x] .
\end{aligned}
$$

We say that a theory $\mathrm{T}_{\mathrm{A}}$ proves well-foundedness of $\prec$ if and only if $\mathrm{T}_{\mathrm{A}} \vdash$ Fund $(\prec, F)$ for every first-order negative formula $F$. Since Fund $(\prec, F)$ also is a negative formula, by Theorem 2 we have that if $\mathrm{T}_{\mathrm{A}}$ proves well-foundedness of $\prec$, then so does $\mathscr{T}$ as well.

§. Realizing subsystems of analysis. In this section we provide realizing terms for additional axioms of analysis listed in the Section 2.

THEOREM 4 (Arithmetic comprehension). Every instance of ACA is realizable in EET.

Proof. Assume a formula $\psi[a] \in \mathscr{L}_{2}$ to be arithmetical. By ECA there exists a term $t$ such that

$$
\mathscr{N}^{\mathrm{N}}[t] \wedge \forall x \varepsilon \text { nat } \forall \mathfrak{x}(\langle x, \mathfrak{x}\rangle \varepsilon t \leftrightarrow \mathfrak{x} \mathbf{r n} \psi[x]) .
$$

We are to prove now that the pair $\langle t, \lambda x .\langle\lambda \mathfrak{x} \cdot \boldsymbol{x}, \lambda \mathfrak{x} \cdot \boldsymbol{x}\rangle\rangle$ is a realization of an instance of $\mathbf{A C A}$

$$
\exists X \forall x(x \in X \leftrightarrow \psi[x]) .
$$

Indeed.

$$
\begin{aligned}
\langle t, \lambda x .\langle\lambda \mathfrak{r} \cdot \mathfrak{x}, \lambda \mathfrak{r} \cdot \mathfrak{x}\rangle\rangle \mathbf{r n} \exists X \forall x(x \in X \leftrightarrow \psi[x]) \\
\quad \equiv \lambda x \cdot\langle\lambda \mathfrak{r} \cdot \mathfrak{x}, \lambda \mathfrak{x} \cdot \mathfrak{x}\rangle \mathbf{r n} \forall x(x \in t \leftrightarrow \psi[x]) \\
\quad \equiv \forall x \varepsilon \text { nat }(\langle\lambda \mathfrak{r} \cdot \mathfrak{x}, \lambda \mathfrak{r} \cdot \mathfrak{x}\rangle \mathbf{r n} x \in t \leftrightarrow \psi[x])) \\
\quad \equiv \forall x \varepsilon \text { nat }(\lambda \mathfrak{r} \cdot \mathfrak{r} \mathbf{r n}(x \in t \rightarrow \psi[x]) \wedge \lambda \mathfrak{r} \cdot \mathfrak{r} \mathbf{r n}(\psi[x] \rightarrow x \in t)),
\end{aligned}
$$

which follows from 4.1 .

THeOREM 5 (Axiom of Choice). Every instance of $\mathbf{A C}$ is realizable in EETJ.

Proof. Assume r rn $\forall x \exists Y \phi[x, Y]$. We then have

$$
\begin{aligned}
& \mathfrak{r} \mathbf{r n} \forall x \\
& \quad \equiv Y \phi[x, Y] \\
& \equiv \forall x \varepsilon \operatorname{nat}\left(\mathfrak{r} x \downarrow \wedge \mathcal{N}^{\mathrm{N}}\left[\mathrm{p}_{0}(\mathfrak{r} x)\right] \wedge \mathrm{p}_{1}(\mathfrak{r} x) \mathbf{r n} \phi\left[x, \mathrm{p}_{0}(\mathfrak{r} x)\right]\right) .
\end{aligned}
$$

By ECA and $\mathbf{J}$ (over nat) let $t:=t[\mathfrak{r}]$ be such that

$$
\mathscr{N}^{\mathrm{N}}[t] \wedge\left(\left\langle\left(x, x_{1}\right), \mathfrak{x}_{1}\right\rangle \varepsilon t \leftrightarrow\left\langle x_{1}, \mathfrak{x}_{1}\right\rangle \varepsilon \mathrm{p}_{0}(\mathfrak{r} x)\right)
$$

Claim. For every formula $\psi[x, Y]$,

$$
\forall x \varepsilon \text { nat } \forall \mathfrak{u}\left(\mathfrak{u} \mathbf{r n} \psi\left[x, \mathrm{p}_{0}(\mathfrak{r} x)\right] \leftrightarrow \mathfrak{u} \mathbf{r n} \psi\left[x, t_{x}\right]\right) .
$$


Proof. First we prove the most important case when $\psi[x . Y]$ is of the form $s \in Y$. We have

$$
\begin{aligned}
\mathfrak{u} \operatorname{rn} s \in t_{x} & \equiv u \operatorname{rn}(x, s) \in t \equiv\langle(x, s), \mathfrak{u}\rangle \varepsilon t \\
& \stackrel{5.4}{=}\langle s, \mathfrak{u}\rangle \varepsilon \mathrm{p}_{0}(\mathfrak{r} x) \equiv \mathfrak{u} \operatorname{rn} s \in \mathrm{p}_{0}(\mathfrak{r} x) .
\end{aligned}
$$

Now, the proof is completed by straightforward induction on $\psi$.

Using this claim, we have, for $x \varepsilon$ nat. $\mathrm{p}_{1}(\mathfrak{r} x) \mathbf{r n} \phi\left[x, t_{x}\right]$. Therefore $\left\langle t[\mathfrak{r}], \lambda x \cdot \mathrm{p}_{1}(\mathfrak{r} x)\right\rangle$ is a realization of the conclusion and $\lambda \mathfrak{r} .\left\langle t[\mathfrak{r}], \lambda x \cdot \mathrm{p}_{1}(\mathfrak{r} x)\right\rangle$ is a realization of the instance of $\mathbf{A C}$

$$
\forall x \exists Y \phi[x, Y] \rightarrow \exists Z \forall x \phi\left[x, Z_{x}\right]
$$

COROllaRY. EHA + ACA + AC is realizable in EETJ and has proof-theoretic strength bounded by $\varphi\left(\varepsilon_{0}, 0\right) .^{2}$

Proof. Realizability follows from Theorems 1, 4 and 5. The bound for prooftheoretic strength follows from the Remarks in the end of Section 3 and the fact $|\mathbf{E E T} \mathbf{J}|=\mid \boldsymbol{\Sigma}_{1}^{1}-\mathbf{A C}$ classical $\mid=\varphi\left(\varepsilon_{0}, 0\right)$ (see, e.g., [6, Section V]).

The following theorem gives an example where $\mathscr{D}$ - and $\mathscr{R}$-interpretations are essentially different. While, according to Theorem $5, \mathbf{T}_{0}$ proves $\mathscr{R}(\mathbf{A C})$, it fails to prove $\mathscr{D}(\mathbf{A C})$; the latter in the presence of the Law of Excluded Middle is at least as strong as full second order arithmetic.

THEOREM $6\left(\mathbf{T}_{0} \nvdash \mathscr{D}(\mathbf{A C})\right)$.

(1) $\mathbf{T}_{0} \nvdash \mathscr{D}(\mathbf{A C})$;

(2) EET + TND $+\mathscr{D}(\mathbf{A C})$ has the strength of at least full analysis.

Proof. Obviously

$$
\text { EHA + ACA + TND is } \mathscr{D} \text {-interpretable in EET + TND }
$$

(see, e.g., $[8$, Section 2]). Then we have

$$
\mathbf{E H A}+\mathbf{A C A}+\mathbf{T N D}+\mathbf{A C} \text { is } \mathscr{D} \text {-interpretable in EET + TND }+\mathscr{D}(\mathbf{A C}) \text {. }
$$

But ACA + TND + AC implies full comprehension, so EHA + ACA + TND + AC is full analysis. By 6.8 we have (2).

For (1), assume $\mathbf{T}_{0} \vdash \mathscr{D}(\mathbf{A C})$. Then $\mathbf{T}_{0}+\mathbf{T N D} \vdash \mathscr{D}(\mathbf{A C})$ and $\mathbf{T}_{0}+\mathbf{T N D}+$ $\mathscr{D}(\mathbf{A C})=\mathbf{T}_{0}+\mathbf{T N D}$. By (2) $\mathbf{T}_{0}+\mathbf{T N D}$ is at least as strong as full analysis, contradiction, since $\mathbf{T}_{0}+$ TND is known to have the strength of $\boldsymbol{\Delta}_{2}^{1}-\mathbf{C A}+\mathbf{B I}$ (classical) (see $[6,10])$.

THEOREM 7 (Replacement). Every instance of $\mathbf{R P}$ is realizable in EETJ.

\footnotetext{
${ }^{2}$ In fact, as shown in [3. Ch. XIII, $\left.\S 2-3\right]$, this bound is exact.
} 
Proof. Assume $\mathscr{N}^{\mathrm{N}}[\alpha]$. Assume also $\left.\mathfrak{r} \mathbf{r n} \forall x \in \alpha \exists ! Y \phi[x, Y]\right)$. By Definition 3.4 we have

$$
\begin{aligned}
& \mathfrak{r} \mathbf{m} \forall x \in \alpha \exists ! Y \phi[x, Y] \\
& \equiv \mathfrak{r} \mathbf{r n} \forall x(x \in \alpha \rightarrow \exists ! Y \phi[x, Y]) \\
& \equiv \forall x \varepsilon \text { nat }(\mathfrak{r} x \downarrow \mathbf{r n}(x \in \alpha \rightarrow \exists ! Y \phi[x, Y])) \\
& \equiv \forall x \in \text { nat }(\mathfrak{r} x \downarrow \wedge \forall \mathfrak{r}(\mathfrak{r} \mathbf{r n} x \in \alpha \rightarrow \mathfrak{r} x \mathfrak{r} \downarrow \mathbf{r n} \exists ! Y \phi[x . Y])) \\
& \equiv \forall x \varepsilon \text { nat }(\mathfrak{r} x \downarrow \wedge \forall \mathfrak{x}(\langle x, \mathfrak{x}\rangle \varepsilon \alpha \rightarrow \mathfrak{r} x \mathfrak{x} \downarrow \mathbf{r n} \exists ! Y \phi[x, Y])) \\
& \equiv \forall x \varepsilon \text { nat }(\mathfrak{r} x \downarrow \wedge \forall \mathfrak{r}(\langle x, \mathfrak{x}\rangle \varepsilon \alpha \\
& \rightarrow \mathfrak{r} x \mathfrak{x} \downarrow \mathbf{r n}(\exists Y(\phi[x, Y] \wedge \forall Z(\phi[x, Z] \rightarrow Y=Z))))) \\
& \equiv \forall x \varepsilon \text { nat }\left(\mathfrak { r } x \downarrow \wedge \forall \mathfrak { x } \left(\langle x , \mathfrak { x } \rangle \varepsilon \alpha \rightarrow \left(\mathfrak{r} x \mathfrak{x} \downarrow \wedge \mathscr{N}^{\mathrm{N}}\left[\mathrm{p}_{0}(\mathfrak{r} x \mathfrak{x})\right]\right.\right.\right. \\
& \left.\left.\left.\wedge \mathrm{p}_{1}(\mathbf{r} x \mathfrak{r}) \mathbf{r n}\left(\phi\left[x, \mathrm{p}_{0}(\mathbf{r} x \mathfrak{r})\right] \wedge \forall Z\left(\phi[x, Z] \rightarrow \mathrm{p}_{0}(\mathfrak{r} x \mathfrak{r})=Z\right)\right)\right)\right)\right) \\
& \equiv \forall x \varepsilon \text { nat }\left(\mathfrak { r } x \downarrow \wedge \forall \mathfrak { r } \left(\langle x , \mathfrak { x } \rangle \varepsilon \alpha \rightarrow \left(\mathscr{N}^{\mathrm{N}}\left[\mathrm{p}_{0}(\mathfrak{r} x \mathfrak{x})\right]\right.\right.\right. \\
& \wedge \mathrm{p}_{0}\left(\mathrm{p}_{1}(\mathfrak{r} x \mathfrak{r})\right) \mathbf{r n} \phi\left[x, \mathrm{p}_{0}(\mathfrak{r} x \mathfrak{x})\right] \\
& \left.\left.\left.\wedge \mathrm{p}_{1}\left(\mathrm{p}_{1}(\mathfrak{r} x \mathfrak{x})\right) \mathbf{r n} \forall Z\left(\phi[x, Z] \rightarrow \mathrm{p}_{0}(\mathfrak{r} x \mathfrak{x})=Z\right)\right)\right)\right) \\
& \equiv \forall x \varepsilon \text { nat }\left(\mathfrak { r } x \downarrow \wedge \forall \mathfrak { x } \left(\langle x , \mathfrak { x } \rangle \varepsilon \alpha \rightarrow \left(\mathscr{N}^{\mathrm{N}}\left[\mathrm{p}_{0}(\mathfrak{r} x \mathfrak{x})\right]\right.\right.\right. \\
& \wedge \mathrm{p}_{0}\left(\mathrm{p}_{1}(\mathfrak{r} \times \mathfrak{r})\right) \text { rn } \phi\left[x, \mathrm{p}_{0}(\mathfrak{r} x \mathfrak{r})\right] \\
& \left.\left.\wedge \forall \gamma\left(\mathscr{N}^{\mathrm{N}}[\gamma] \rightarrow\left(\mathbf{p}_{1}\left(\mathbf{p}_{1}(\mathfrak{r} x \mathfrak{x})\right) \gamma \downarrow \mathbf{r n}\left(\phi[x, \gamma] \rightarrow \mathbf{p}_{0}(\mathfrak{r} x \mathfrak{x})=\gamma^{\prime}\right)\right)\right)\right)\right) \\
& \equiv \forall x \varepsilon \text { nat }\left(\mathfrak { r } x \downarrow \wedge \forall \mathfrak { r } \left(\langle x , \mathfrak { x } \rangle \varepsilon \alpha \rightarrow \left(\mathscr{N}^{\mathrm{N}}\left[\mathrm{p}_{0}(\mathfrak{r} x \mathfrak{x})\right]\right.\right.\right. \\
& \left.\wedge \mathrm{p}_{0}\left(\mathrm{p}_{1}(\mathrm{r} x \mathfrak{x})\right) \mathbf{r n} \phi\left[x, \mathrm{p}_{0}(\mathfrak{r} x \mathfrak{x})\right]\right) \wedge \forall \gamma\left(\mathscr { N } ^ { N } [ \gamma ] \rightarrow \left(\mathrm{p}_{1}\left(\mathrm{p}_{1}(\mathfrak{r} x \mathfrak{x})\right) \gamma \downarrow\right.\right. \\
& \left.\left.\left.\left.\left.\wedge \forall \mathfrak{q}\left(\mathbf{q} \mathbf{r n} \phi[x, \gamma] \rightarrow \mathrm{p}_{1}\left(\mathbf{p}_{1}(\mathfrak{r} x \mathfrak{r})\right) \gamma \mathfrak{q} \downarrow \mathbf{r n} \mathbf{p}_{0}(\mathbf{r} x \mathfrak{r})=\gamma\right)\right)\right)\right)\right)\right) \text {. }
\end{aligned}
$$

Continuing 7.9 .

$$
\begin{aligned}
& \mathfrak{r}_{1} \text { rn } \mathrm{p}_{0}(\mathfrak{r} x \mathfrak{x})=\gamma \\
& \equiv \mathfrak{r}_{1} \mathbf{r n} \forall x_{1}\left(\left(x_{1} \in \mathrm{p}_{0}(\mathfrak{r} x \mathfrak{x}) \rightarrow x_{1} \in \gamma\right) \wedge\left(x_{1} \in \gamma \rightarrow x_{1} \in \mathrm{p}_{0}(\mathfrak{r} x \mathfrak{x})\right)\right) \\
& \equiv \forall x_{1} \varepsilon \text { nat }\left(\mathfrak { r } _ { 1 } x _ { 1 } \downarrow \wedge \left(\forall \mathfrak{x}_{0}\left(\left\langle x_{1}, \mathfrak{x}_{0}\right\rangle \varepsilon \mathrm{p}_{0}(\mathfrak{r} x \mathfrak{x}) \rightarrow\left\langle x_{1}, \mathrm{p}_{0}\left(\mathfrak{r}_{1} x_{1}\right) \mathfrak{x}_{0}\right\rangle \varepsilon \gamma\right)\right.\right. \\
& \left.\left.\wedge \forall \mathfrak{x}_{1}\left(\left\langle x_{1}, \mathfrak{x}_{1}\right\rangle \varepsilon \gamma \rightarrow\left\langle x_{1}, \mathrm{p}_{1}\left(\mathfrak{r}_{1} x_{1}\right) \mathfrak{x}_{1}\right\rangle \varepsilon \mathrm{p}_{0}(\mathfrak{r} x \mathfrak{r})\right)\right)\right) .
\end{aligned}
$$

Also,

$$
\begin{aligned}
& \mathfrak{u} \mathbf{r n} \forall x \in \alpha \phi\left[x, C_{x}\right] \\
& \quad \equiv \mathfrak{u} \mathbf{r n} \forall x\left(x \in \alpha \rightarrow \phi\left[x, C_{x}\right]\right) \\
& \quad \equiv \forall x \varepsilon \text { nat }\left(\mathfrak{u} x \downarrow \mathbf{r n}\left(x \in \alpha \rightarrow \phi\left[x, C_{x}\right]\right)\right) \\
& \quad \equiv \forall x \in \text { nat }\left(u x \downarrow \wedge \forall \mathfrak{x}\left(\langle x, \mathfrak{x}\rangle \in \alpha \rightarrow \mathfrak{u} x \mathfrak{x} \downarrow \mathbf{r n} \phi\left[x, C_{x}\right]\right)\right) .
\end{aligned}
$$

By ECA there exists a term $t:=t[\alpha, \mathfrak{r}]$ such that

$$
\begin{array}{r}
\mathscr{N}^{N}[t[\alpha, \mathfrak{r}]] \wedge\left(\left\langle\left(x, x_{1}\right), \mathfrak{y}\right\rangle \varepsilon t[\alpha, \mathfrak{r}] \leftrightarrow \mathfrak{y}=\left\langle\mathrm{p}_{0} \mathfrak{y}, \mathrm{p}_{1} \mathfrak{y}\right\rangle\right. \\
\left.\wedge\left\langle\left\langle x, \mathrm{p}_{0} \mathfrak{y}\right\rangle,\left\langle x_{1}, \mathrm{p}_{1} \mathfrak{y}\right\rangle\right\rangle \varepsilon \mathrm{j}\left(\alpha, \lambda y \cdot \mathrm{p}_{0}\left(\mathfrak{r}\left(\mathrm{p}_{0} y\right)\left(\mathrm{p}_{1} y\right)\right)\right)\right)
\end{array}
$$


If we had $\langle x, \mathfrak{x}\rangle \varepsilon \alpha \rightarrow\left(\mathfrak{r}_{2} \mathbf{r n} \phi\left[x, \mathrm{p}_{0}(\mathfrak{r} x \mathfrak{x})\right] \leftrightarrow \mathfrak{r}_{2} \mathbf{r n} \phi\left[x, t_{x}\right]\right)$, this would provide us with a realization of the Axiom of Choice on $\alpha$ (instead of Replacement) (cf. the proof of Theorem 5). While this is not the case. by making use of uniqueness part of 7.9 we obtain a pair of operations, which map realizations of $\phi\left[x, p_{0}(\mathfrak{r} x \mathfrak{x})\right]$ and $\phi\left[x . t_{x}\right]$ into each other. This pair of operations is represented by a term $\mathfrak{r}_{\phi}^{Y}$ defined below. and is sufficient to build up a realization of Replacement.

For each formula $\varphi:=\varphi[x, Y] \in \mathscr{L}_{2}$ such that $\mathfrak{x} \notin \mathrm{FV}_{0}(\varphi)$ and a predicate variable $Y \in \mathscr{L}_{2}$ we define a term $\mathfrak{r}_{\varphi}^{Y}:=\mathbf{r}_{\varphi}^{Y}\left[\mathfrak{r}, x, \mathfrak{x}, \mathrm{FV}_{0}(\varphi)\right]$ by recursion on $\varphi$ in the following way:

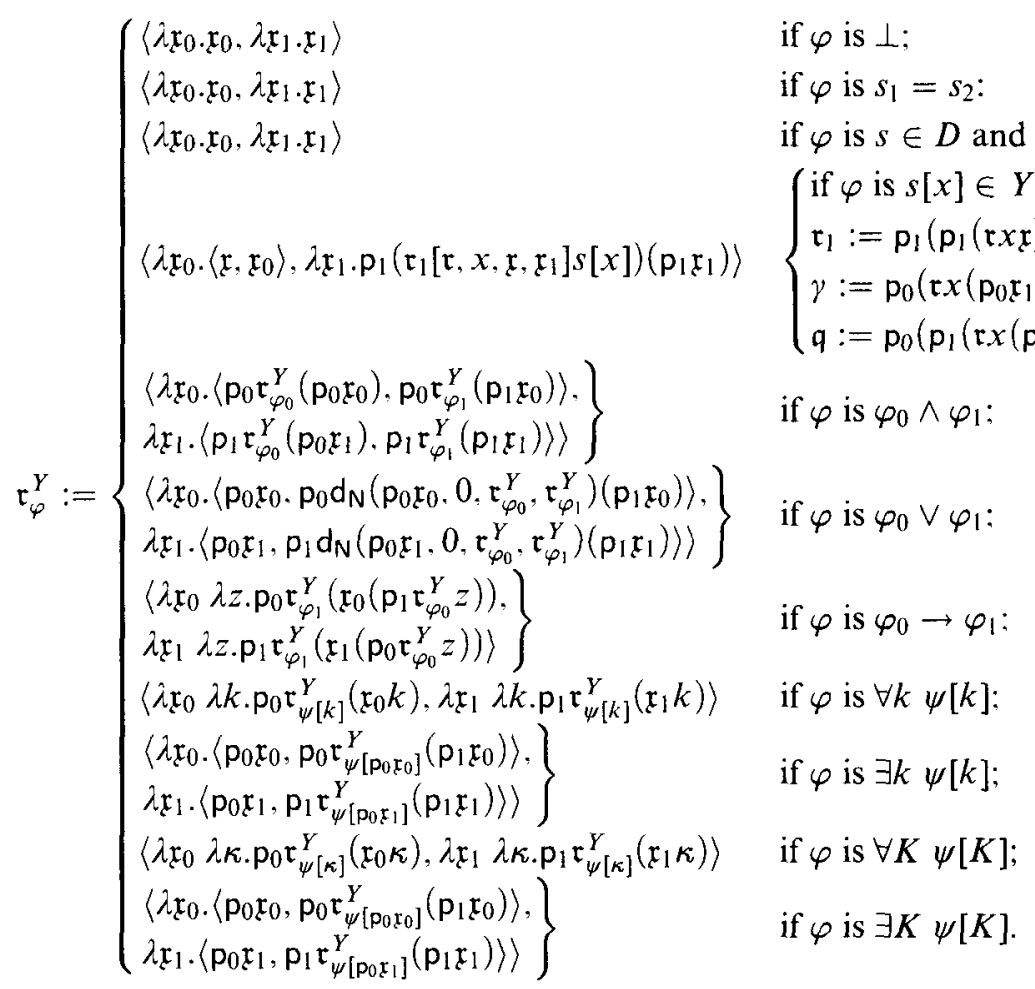

Claim. For every formula $\varphi:=\varphi[x, Y] \in \mathscr{L}_{2}$ we have

$$
\langle x, \mathfrak{x}\rangle \varepsilon \alpha \rightarrow \mathfrak{r}_{\varphi}^{Y}[\mathfrak{r}, x, \mathfrak{r}] \mathbf{r n}\left(\varphi\left[x, \mathbf{p}_{0}(\mathfrak{r} x \mathfrak{r})\right] \leftrightarrow \varphi\left[x, t_{x}\right]\right) .
$$

Proof. We check only the most important case (the only one which requires use of uniqueness in the premise of Replacement axiom), when $\varphi$ is of the form $s[x] \in Y$. Other cases are proved by routine induction on $\varphi$.

By Definition 3.4 we have

$$
\begin{gathered}
\mathfrak{x}_{0} \mathbf{r n} s[x] \in \mathrm{p}_{0}(\mathfrak{r} x \mathfrak{x}) \equiv\left\langle s[x], \mathfrak{x}_{0}\right\rangle \varepsilon \mathbf{p}_{0}(\mathfrak{r} x \mathfrak{x}), \\
\mathfrak{x}_{1} \mathbf{r n} s[x] \in t_{x} \equiv \mathfrak{x}_{1} \mathbf{r n}(x, s[x]) \in t \equiv\left\langle(x, s[x]), \mathfrak{x}_{1}\right\rangle \varepsilon t \\
\stackrel{7.12}{\Longleftrightarrow}\left\langle\left\langle x, \mathrm{p}_{0} \mathfrak{x}_{1}\right\rangle,\left\langle s[x], \mathbf{p}_{1} \mathfrak{x}_{1}\right\rangle\right\rangle \varepsilon \mathbf{j}\left(\alpha, \lambda y \cdot \mathbf{p}_{0}\left(\mathbf{r}\left(\mathrm{p}_{0} y\right)\left(\mathbf{p}_{1} y\right)\right)\right) .
\end{gathered}
$$


By $\mathbf{J} 7.14$ and 7.15 immediately yield

$$
\langle x, \mathfrak{x}\rangle \varepsilon \alpha \rightarrow \lambda \mathfrak{x}_{0} .\left\langle\mathfrak{x}, \mathfrak{x}_{0}\right\rangle \mathbf{r n}\left(s[x] \in \mathbf{p}_{0}(\mathfrak{r} x \mathfrak{x}) \rightarrow s[x] \in t_{x}\right) .
$$

For the opposite direction, assume $\langle x, \mathfrak{x}\rangle \in \alpha$ and $\mathfrak{x}_{1} \mathbf{r n} s[x] \in t_{x}$, i.e.,

$$
\left\langle\left\langle x, \mathrm{p}_{0} \mathfrak{x}_{1}\right\rangle,\left\langle s[x], \mathrm{p}_{1} \mathfrak{r}_{1}\right\rangle\right\rangle \varepsilon j\left(\alpha, \lambda y \cdot \mathrm{p}_{0}\left(\mathfrak{r}\left(\mathrm{p}_{0} y\right)\left(\mathrm{p}_{1} y\right)\right)\right) .
$$

Then by $\mathbf{J}$

$$
\left\langle x, \mathrm{p}_{0} \mathfrak{r}_{1}\right\rangle \varepsilon \alpha \wedge\left\langle s[x], \mathrm{p}_{1} \mathfrak{r}_{1}\right\rangle \varepsilon \mathrm{p}_{0}\left(\mathfrak{r} x\left(\mathrm{p}_{0} \mathfrak{x}_{1}\right)\right) .
$$

By 7.9 we have

$$
\mathscr{N}^{N}\left[\mathrm{p}_{0}\left(\mathfrak{r} x\left(\mathrm{p}_{0} \mathfrak{x}_{1}\right)\right)\right] \wedge \mathrm{p}_{0}\left(\mathrm{p}_{1}\left(\mathfrak{r} x\left(\mathrm{p}_{0} \mathfrak{x}_{1}\right)\right)\right) \mathbf{r n} \phi\left[x, \mathrm{p}_{0}\left(\mathfrak{r} x\left(\mathrm{p}_{0} \mathfrak{x}_{1}\right)\right)\right] .
$$

Now by the uniqueness part of 7.9 we obtain

$$
\mathrm{p}_{1}\left(\mathrm{p}_{1}(\mathfrak{r} x \mathfrak{x})\right) \gamma \mathfrak{q} \downarrow \mathbf{r n} \mathrm{p}_{0}(\mathfrak{r} x \mathfrak{r})=\gamma,
$$

where $\gamma:=\mathrm{p}_{0}\left(\mathfrak{r} x\left(\mathrm{p}_{0} \mathfrak{x}_{1}\right)\right)$ and $\mathfrak{q}:=\mathrm{p}_{0}\left(\mathrm{p}_{1}\left(\mathfrak{r} x\left(\mathrm{p}_{0} \mathfrak{x}_{1}\right)\right)\right)$. Taking

$$
\mathfrak{r}_{1}:=\mathrm{p}_{1}\left(\mathrm{p}_{1}(\mathfrak{r} x \mathfrak{r})\right) \gamma\left[\mathfrak{r}, x, \mathfrak{x}_{1}\right] \mathfrak{q}\left[\mathfrak{r}, x, \mathfrak{x}_{1}\right],
$$

from 7.10 and 7.17 we have

$$
\left\langle s[x], \mathrm{p}_{1}\left(\mathfrak{r}_{1} s[x]\right)\left(\mathbf{p}_{1} \mathfrak{x}_{1}\right)\right\rangle \varepsilon \mathbf{p}_{0}(\mathfrak{r} x \mathfrak{x}) .
$$

Equation 7.20 shows that

$$
\begin{gathered}
\langle x, \mathfrak{x}\rangle \varepsilon \alpha \rightarrow \lambda \mathfrak{x}_{1} \cdot \mathrm{p}_{1}\left(\mathfrak{r}_{1}\left[\mathfrak{r}, x, \mathfrak{x}, \mathfrak{x}_{1}\right] s[x]\right)\left(\mathrm{p}_{1} \mathfrak{x}_{1}\right) \\
\operatorname{rn}\left(s[x] \in t_{x} \rightarrow s[x] \in \mathrm{p}_{0}(\mathfrak{r} x \mathfrak{x})\right),
\end{gathered}
$$

The previous claim and equation 7.9 together prove that

$$
\langle x, \mathfrak{x}\rangle \varepsilon \alpha \rightarrow\left(\mathbf{p}_{0} \mathfrak{r}_{\phi[x, Y]}^{Y}[\mathfrak{r}, x, \mathfrak{x}]\left(\mathbf{p}_{0}\left(\mathbf{p}_{1}(\mathfrak{r} x \mathfrak{x})\right)\right) \downarrow \mathbf{r n} \phi\left[x,(t[\alpha, \mathfrak{r}])_{x}\right]\right),
$$

which is to say that

$$
\begin{aligned}
& \mathfrak{r} \mathbf{r n} \forall x \in \alpha \exists ! Y \phi[x, Y] \\
& \quad \rightarrow \lambda x \lambda \mathfrak{r} \cdot \mathrm{p}_{0} \mathfrak{r}_{\phi[x, Y]}^{Y}[\mathfrak{r}, x, \mathfrak{x}]\left(\mathrm{p}_{0}\left(\mathrm{p}_{1}(\mathfrak{r} x \mathfrak{r})\right)\right) \mathbf{r n} \forall x \in \alpha \phi\left[x,(t[\alpha, \mathfrak{r}])_{x}\right] .
\end{aligned}
$$

The last equation shows that an operation

$$
\lambda \alpha \lambda \mathfrak{r} .\left\langle t[\alpha, \mathfrak{r}], \lambda x \lambda \mathfrak{r} \cdot \mathrm{p}_{0} \mathfrak{r}_{\phi[x, Y]}^{Y}[\mathfrak{r}, x, \mathfrak{r}]\left(\mathrm{p}_{0}\left(\mathrm{p}_{1}(\mathfrak{r} x \mathfrak{r})\right)\right)\right\rangle
$$

is a realization for an instance of $\mathbf{R P}$

$$
\forall X\left(\forall x \in X \exists ! Y \phi[x, Y] \rightarrow \exists Z \forall x \in X \phi\left[x, Z_{x}\right]\right)
$$

THEOREM 8 (Inductive Generation). Every instance of IGA is realizable in EET + IG.

Proof. Assume $\mathscr{N}^{\mathrm{N}}[\alpha] \wedge \mathscr{N}^{\mathrm{N}}[\beta]$. Assume also

$$
\mathfrak{r} \operatorname{rn} \forall x \varepsilon \alpha\left(\forall y\left(y<_{\beta} x \rightarrow \phi[y]\right) \rightarrow \phi[x]\right) .
$$


By Definition 3.4 we have:

$$
\begin{aligned}
\mathfrak{r} \mathbf{r n} \forall x \varepsilon \alpha\left(\forall y\left(y<_{\beta} x \rightarrow \phi[y]\right) \rightarrow \phi[x]\right) \\
\equiv \mathfrak{r} \mathbf{r n} \forall x\left(x \varepsilon \alpha \rightarrow\left(\forall y\left(y<_{\beta} x \rightarrow \phi[y]\right) \rightarrow \phi[x]\right)\right) \\
\equiv \forall x \varepsilon \operatorname{nat}(\mathfrak{r} x \downarrow \wedge \forall \mathfrak{r}(\langle x, \mathfrak{x}\rangle \varepsilon \alpha \rightarrow \\
\left.\left.\quad \mathfrak{r} x \mathfrak{r} \downarrow \mathbf{r n}\left(\forall y\left(y<_{\beta} x \rightarrow \phi[y]\right) \rightarrow \phi[x]\right)\right)\right) \\
\equiv \forall x \varepsilon \operatorname{nat}(\mathfrak{r} x \downarrow \wedge \forall \mathfrak{r}(\langle x, \mathfrak{x}\rangle \varepsilon \alpha \rightarrow(\mathfrak{r} x \mathfrak{r} \downarrow \\
\left.\left.\left.\quad \wedge \forall \mathfrak{u}\left(\mathfrak{u} \mathbf{r n} \forall y\left(y<_{\beta} x \rightarrow \phi[y]\right) \rightarrow \mathfrak{r} x \mathfrak{x u} \downarrow \mathbf{r n} \phi[x]\right)\right)\right)\right) \\
\equiv \forall x \varepsilon \operatorname{nat}(\mathfrak{r} x \downarrow \wedge \forall \mathfrak{r}(\langle x, \mathfrak{x}\rangle \varepsilon \alpha \rightarrow(\mathfrak{r} x \mathfrak{r} \downarrow \wedge \forall \mathfrak{u}(\forall y \varepsilon \text { nat }(\mathfrak{u} y \downarrow \\
\quad \forall \mathfrak{v}(\mathfrak{v} \mathbf{r n}(y, x) \varepsilon \beta \rightarrow \mathfrak{u} y \mathfrak{v} \downarrow \mathbf{r n} \phi[y])) \rightarrow \mathfrak{r} x \mathfrak{r} \mathfrak{u} \downarrow \mathbf{r n} \phi[x])))) \\
\equiv \forall x \varepsilon \operatorname{nat}(\mathfrak{r} x \downarrow \wedge \forall \mathfrak{x}(\langle x, \mathfrak{x}\rangle \varepsilon \alpha \rightarrow(\mathfrak{r} x \mathfrak{x} \downarrow \wedge \forall \mathfrak{u}(\forall y \varepsilon \text { nat }(\mathfrak{u} y \downarrow \\
\wedge \forall \mathfrak{v}(\langle(y, x) \cdot \mathfrak{v}\rangle \varepsilon \beta \rightarrow \mathfrak{u} y \mathfrak{v} \downarrow \mathbf{r n} \phi[y])) \rightarrow \mathfrak{r} x \mathfrak{r} \mathfrak{r} \downarrow \mathbf{r n} \phi[x])))) .
\end{aligned}
$$

By recursion theorem for a function $f:=\lambda z \lambda x \lambda \mathfrak{r} \cdot \mathbf{x} \mathfrak{x}(\lambda y \lambda \mathfrak{y} . z y \mathfrak{y})$ there exists a term $\mathrm{R}:=\operatorname{rec} f$ such that

$$
\mathrm{R} x \mathfrak{x} \simeq \mathfrak{r} x \mathfrak{r}(\lambda y \lambda \mathfrak{v} . \mathrm{R} y \mathfrak{v}) .
$$

By ECA there exists a term $\beta^{*}:=\beta^{*}[\beta]$ such that

$$
\begin{aligned}
\mathscr{N}\left[\beta^{*}\right] & \wedge\left(u \varepsilon \beta^{*} \leftrightarrow u=\left\langle\mathrm{p}_{0} u, \mathrm{p}_{1} u\right\rangle\right. \\
& \wedge \mathrm{p}_{0} u=\left\langle\mathrm{p}_{0} \mathrm{p}_{0} u, \mathrm{p}_{1} \mathrm{p}_{0} u\right\rangle \wedge \mathrm{p}_{1} u=\left\langle\mathrm{p}_{0} \mathrm{p}_{1} u, \mathrm{p}_{1} \mathrm{p}_{1} u\right\rangle \\
& \left.\wedge \mathrm{p}_{0} \mathrm{p}_{0} u \varepsilon \text { nat } \wedge \mathrm{p}_{0} \mathrm{p}_{1} u \varepsilon \text { nat } \wedge\left\langle\left(\mathrm{p}_{0} \mathrm{p}_{0} u, \mathrm{p}_{0} \mathrm{p}_{1} u\right), \mathrm{p}_{1} \mathrm{p}_{0} u\right\rangle \varepsilon \beta\right)
\end{aligned}
$$

We want to prove $\operatorname{Prog}_{\beta^{*}}(\alpha, \operatorname{Ry} \mathfrak{v} \mathbf{r n} \phi[y])$, i.e.,

$$
\begin{aligned}
& \langle x, \mathfrak{x}\rangle \varepsilon \alpha \rightarrow(\forall y \varepsilon \text { nat } \forall \mathfrak{v} \\
& \left.\quad\left(\langle\langle y, \mathfrak{v}\rangle,\langle x, \mathfrak{x}\rangle\rangle \varepsilon \beta^{*} \rightarrow \operatorname{R} y \mathfrak{v} \downarrow \mathbf{r n} \phi[y]\right) \rightarrow \operatorname{R} x \mathfrak{x} \downarrow \mathbf{r n} \phi[x]\right) .
\end{aligned}
$$

Assume $\langle x, \mathfrak{x}\rangle \varepsilon \alpha \wedge \forall y \varepsilon$ nat $\forall \mathfrak{v}\left(\langle\langle y, \mathfrak{v}\rangle,\langle x, \mathfrak{x}\rangle\rangle \varepsilon \beta^{*} \rightarrow \operatorname{R} y \mathfrak{v} \downarrow \mathbf{r n} \phi[y]\right)$. Then by 8.26 we have

$$
\forall y \varepsilon \text { nat } \forall \mathfrak{v}(\langle(y, x), \mathfrak{v}\rangle \varepsilon \beta \rightarrow \mathrm{R} y \mathfrak{v} \downarrow \mathbf{r n} \phi[y]) .
$$

Therefore for the operation $\mathfrak{u}:=\lambda y \lambda \mathfrak{v} . \operatorname{Ryv}$ by 8.24 we have $\mathfrak{r} x \mathfrak{r} u \downarrow$ rn $\phi[x]$, i.e.. $\mathfrak{r} x \mathfrak{r}(\lambda y \lambda \mathfrak{v} . \operatorname{Ryv}) \downarrow \mathbf{r n} \phi[x]$. From this fact and equation 8.25 we obtain 8.27.

By IG we obtain

$$
\forall x \varepsilon \text { nat } \forall \mathfrak{x}\left(\langle x, \mathfrak{x}\rangle \varepsilon \mathrm{i}\left(\alpha, \beta^{*}\right) \rightarrow \mathrm{R} x \mathfrak{x} \downarrow \mathbf{r n} \phi[x]\right),
$$

or

$$
\lambda x \lambda \mathfrak{x} \cdot \operatorname{Rx} \mathfrak{r} \mathbf{r n} \forall x \in i\left(\alpha, \beta^{*}\right) \phi[x] .
$$

This shows that an operation $\lambda \mathfrak{r} \lambda x \lambda \mathfrak{r}$. Rxx is a realization of a "part" of IGA

$$
\forall x \varepsilon \alpha\left(\forall y\left(y<_{\beta} x \rightarrow \phi[y]\right) \rightarrow \phi[x]\right) \rightarrow \forall z \varepsilon \mathrm{i}\left(\alpha, \beta^{*}\right) \phi[z] .
$$


Applying the above considerations to the formula $x \in U$ in place of $\phi[x]$, we see that $\lambda \gamma \lambda \mathfrak{r} \lambda x \lambda \mathfrak{r}$. Rxx is a realization of

$$
\forall U\left(\operatorname{Prog}_{\beta}[\alpha, U] \rightarrow \mathrm{i}\left(\alpha, \beta^{*}\right) \subseteq U\right),
$$

Last. we have to find a realization of

$$
\operatorname{Prog}_{\beta}\left[\alpha . i\left(\alpha, \beta^{*}\right)\right]
$$

By 8.24 with $x \in \mathrm{i}\left(\alpha . \beta^{*}\right)$ in place of $\phi[x]$, we have

$$
\begin{aligned}
& q \text { rn } \operatorname{Prog}_{\beta}\left[\alpha, \mathrm{i}\left(\alpha, \beta^{*}\right)\right] \\
& \equiv \mathfrak{q} \mathbf{r n} \forall x \varepsilon \alpha\left(\forall y\left(y<_{\beta} x \rightarrow y \in \mathrm{i}\left(\alpha, \beta^{*}\right)\right) \rightarrow x \in \mathrm{i}\left(\alpha, \beta^{*}\right)\right) \\
& \equiv \forall x \in \text { nat }(\mathfrak{q} x \downarrow \wedge \forall \mathfrak{x}(\langle x, \mathfrak{x}\rangle \varepsilon \alpha \rightarrow(\mathfrak{q} x \mathfrak{x} \downarrow \wedge \forall \mathfrak{u}(\forall y \varepsilon \text { nat } \\
& \left(\mathfrak{u} y \downarrow \wedge \forall \mathfrak{v}\left(\langle(y, x), \mathfrak{v}\rangle \varepsilon \beta \rightarrow \mathfrak{u} y \mathfrak{v} \downarrow \text { rn } y \in \mathrm{i}\left(\alpha, \beta^{*}\right)\right)\right) \rightarrow \\
& \left.\left.\left.\left.\mathfrak{q} x \mathfrak{x u} \downarrow \mathbf{r n} x \in \mathbf{i}\left(\alpha, \beta^{*}\right)\right)\right)\right)\right) \\
& \equiv \forall x \varepsilon \text { nat }(\mathfrak{q} x \downarrow \wedge \forall \mathfrak{x}(\langle x, \mathfrak{x}\rangle \varepsilon \alpha \rightarrow(\mathfrak{q} x \mathfrak{x} \downarrow \wedge \forall \mathfrak{u}(\forall y \varepsilon \text { nat } \\
& \left(\mathfrak{u} y \downarrow \wedge \forall \mathfrak{v}\left(\langle(y, x), \mathfrak{v}\rangle \varepsilon \beta \rightarrow\langle y, \mathfrak{u} y \mathfrak{v}\rangle \varepsilon \mathrm{i}\left(\alpha, \beta^{*}\right)\right)\right) \rightarrow \\
& \left.\left.\left.\left.\langle x, \mathfrak{q} x \mathfrak{r} \mathfrak{u}\rangle \varepsilon \mathrm{i}\left(\alpha, \beta^{*}\right)\right)\right)\right)\right) \\
& \stackrel{8.26}{\Longleftrightarrow} \forall x \varepsilon \text { nat }(\mathfrak{q} x \downarrow \wedge \forall \mathfrak{x}(\langle x, \mathfrak{x}\rangle \varepsilon \alpha \rightarrow(\mathfrak{q} x \mathfrak{x} \downarrow \wedge \forall \mathfrak{u}(\forall y \varepsilon \text { nat } \\
& \left(\mathfrak{u} y \downarrow \wedge \forall \mathfrak{v}\left(\langle\langle y, \mathfrak{v}\rangle,\langle x, \mathfrak{x}\rangle\rangle \varepsilon \beta^{*} \rightarrow\langle y, \mathfrak{u} y \mathfrak{v}\rangle \varepsilon \mathrm{i}\left(\alpha, \beta^{*}\right)\right)\right) \rightarrow \\
& \left.\left.\left.\left.\langle x, \mathfrak{q} x \mathfrak{w} \mathfrak{u}\rangle \in \mathrm{i}\left(\alpha, \beta^{*}\right)\right)\right)\right)\right) .
\end{aligned}
$$

Assume now $\langle x, \mathfrak{x}\rangle \varepsilon \alpha \wedge \forall y \varepsilon$ nat $\left(\mathfrak{u} y \downarrow \wedge \forall \mathfrak{v}\left(\langle\langle y, \mathfrak{v}\rangle,\langle x, \mathfrak{x}\rangle\rangle \varepsilon \beta^{*} \rightarrow\langle y, \mathfrak{u} y \mathfrak{v}\rangle \varepsilon\right.\right.$ $\left.\left.\mathrm{i}\left(\alpha, \beta^{*}\right)\right)\right)$. Note that from Definition 8.26 of $\beta^{*}$ it follows that

$$
\begin{aligned}
& \exists \mathfrak{v}\left(\left\langle\left\langle y_{0}, \mathfrak{v}_{0}\right\rangle,\left\langle y_{1}, \mathfrak{v}\right\rangle\right\rangle \varepsilon \beta^{*}\right) \\
& \quad \leftrightarrow \forall \mathfrak{v}\left(\left\langle\left\langle\left\langle y_{0}, \mathfrak{v}_{0}\right\rangle,\left\langle y_{1}, \mathfrak{v}\right\rangle\right\rangle \varepsilon \beta^{*}\right)\right.
\end{aligned}
$$

and therefore by IG

$$
\begin{aligned}
& \exists \mathfrak{v}\left(\langle y, \mathfrak{v}\rangle \varepsilon \mathrm{i}\left(\alpha, \beta^{*}\right)\right) \\
& \quad \leftrightarrow \forall \mathfrak{v}\left(\langle y, \mathfrak{v}\rangle \varepsilon \mathrm{i}\left(\alpha, \beta^{*}\right)\right) .
\end{aligned}
$$

So, we also have

$$
\forall y \varepsilon \text { nat } \forall \mathfrak{v}\left(\langle\langle y, \mathfrak{v}\rangle,\langle x, \mathfrak{x}\rangle\rangle \varepsilon \beta^{*} \rightarrow\langle y, \mathfrak{v}\rangle \varepsilon \mathrm{i}\left(\alpha, \beta^{*}\right)\right) .
$$

By IG we obtain $\langle x, \mathfrak{x}\rangle \varepsilon \mathrm{i}\left(\alpha, \beta^{*}\right)$. This demonstrates that an operation $\mathfrak{q}:=\lambda x \lambda \mathfrak{x} \lambda \mathbf{u} \cdot \mathfrak{x}$ is a realization of 8.33 .

Bringing realizations of $8.31-8.33$ together shows that an operation

$$
\left.\lambda \alpha \lambda \beta \text {. }\left\langle i\left(\alpha, \beta^{*}[\beta]\right) .(\langle\lambda x \lambda \mathfrak{r} \lambda \mathfrak{u} . \mathfrak{x} . \lambda \gamma \lambda \mathfrak{r} \lambda x \lambda \mathfrak{r} . \mathrm{R} x \mathfrak{x}\rangle, \lambda \tau \lambda x \lambda \mathfrak{r} . \mathrm{R} x \mathfrak{x}\rangle\right\rangle\right)
$$

is a realization of an instance of IGA

$$
\begin{aligned}
& \forall X \forall Y \exists Z\left(\mathrm{WP}_{Y}[X, Z] \wedge(\forall x \in X\right. \\
& \left.\left.\quad\left(\forall y\left(y<_{Y} x \rightarrow \phi[y]\right) \rightarrow \phi[x]\right) \rightarrow \forall z \in Z \phi[z]\right)\right) .
\end{aligned}
$$

COROLLARY. IARI is realizable in $\mathbf{T}_{0}$; its proof-theoretic strength is bounded by that of $\mathbf{T}_{0}$.

Proof. This follows from Theorems 1.4. 7 and 8. 


\section{REFERENCES}

[1] P. ACZEL. The type theoretic interpretation of constructive set theory, Logic colloquium '77 (A. MacIntyre, L. Pacholski, and J. Paris, editors), 1978, pp. 55-66.

[2] — The type theoretic interpretation of constructive set theory: inductive definitions. Logic, methodology and philosophy of science vii (R. B. Marcus et al., editors). Amsterdam. 1986.

[3] M. BEESON. Foundations of constructive mathematics. Springer-Verlag. 1985.

[4] W. Buchholz. S. Feferman, W. Pohlers. and W. Sieg. Iterated inductive definitions and subsystems of analysis, Lecture Notes in Mathematics, vol. 897, Springer-Verlag, 1981.

[5] S. Feferman, A language and axioms for explicit mathematics, Algebra and logic, Lecture Notes in Mathematics, vol. 450, Springer-Verlag, 1975, pp. 87-139.

[6] — Constructive theories of functions and classes, Logic colloquium '78. 1979, pp. 159-224.

[7] S. FEFERMAN and G. JÄGER, Systems of explicit mathematic's with non-constructive $\mu$-operator. Part II, Annals of Pure and Applied Logic, vol. 79 (1996), no. 1, pp. 37-52.

[8] T. GLAss, Standardstrukturen für Systeme Expliziter Mathematik, Ph. D. Dissertation. Münster. 1993.

[9] E. GRIFFor and M. RATHJEN, The strength of some Martin-Lö type theories. Archive for Mathematical Logic, vol. 33 (1994), pp. 347-385.

[10] G. JÄGER, A well-ordering proof for Feferman's theory $\boldsymbol{T}_{0}$, Archive for Mathematical Logic, vol. 23 (1983), pp. 65-77.

[11] - Induction in elementary theory of types and names. Computer science logic '87. LNCS, vol. 329. Springer-Verlag, 1988, pp. 118-128.

[12] G. JÄGER, R. KAHLE, and T. STRAHM, On applicative theories, Logic and foundations of mathematics (A. Cantini, E. Casari, and P. L. Minari, editors), 1999, pp. 83-92.

[13] G. JäGER and W. POHLERS, Eine beweistheoretische Untersuchung von $\Delta_{2}^{1}-\mathrm{CA}+\mathrm{BI}$ und verwandter Systeme, Sitz. Beyer. Akad. der Wissen., Math.-Natur. Klasse, (1982), pp. 1-28.

[14] M. RATHJEN, Interpreting Mahlo set theory in Mahlo type theory, preprint. 1999.

[15] M. Rathien, E. R. Griffor, and E. Palmgren, Inaccessibility in constructive set theory and type theory, Annals of Pure and Applied Logic, vol. 94 (1998), pp. 181-200.

[16] A. SETZER, Well-ordering proof for Martin-Löf type theory with W-type and one universe, Annals of Pure and Applied Logic, vol. 92 (1998), pp. 113-159.

[17] M. TATSUTA. Realizability for constructive theory of functions and classes and its application to program synthesis, Proceedings of thirteenth annual IEEE symposium on logic in computer science. 1998. pp. $358-367$.

[18] A. Troelstra, Realizability, Handbook of proof theory (S. Buss, editor), North-Holland. 1998. pp. $407-474$.

[19] A. Troelstra and D. van Dallen, Constructivism in mathematics, vol. I, North-Holland, 1988.

[20] S. TUPAILO, Realization of constructive set theory into explicit mathematics: a lower bound for impredicative Mahlo universe, Technical report IAM-00-004. University of Bern. Switzerland, submitted for publication.

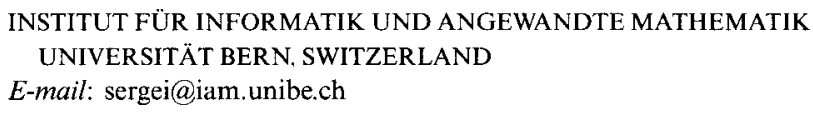

